\title{
CORRIGENDUM
}

\section{Turning aluminium into a noble-metal-like catalyst for low-temperature activation of molecular hydrogen}

Irinder S. Chopra, Santanu Chaudhuri, Jean François Veyan and Yves J. Chabal

Nature Materials 10, 884-889 (2011); published online 25 September 2011; corrected after print 21 October 2011.

In the version of this Article originally published, the first sentence of the Acknowledgements should have read that the work performed at the University of Texas at Dallas was fully supported by the Office of Basic Energy Sciences, US Department of Energy, under contract no. DE-AC02-98CH10886. This error has been corrected in the HTML and PDF versions of the Article. 